\title{
Association of microRNAs and pathologic response to preoperative chemotherapy in triple negative breast cancer: preliminary report
}

\author{
Agnieszka Kolacinska - Jan Morawiec - Wojciech Fendler • Beata Malachowska • \\ Zbigniew Morawiec · Janusz Szemraj • Zofia Pawlowska • Dipanjan Chowdhury • \\ Young Eun Choi $\cdot$ Robert Kubiak $\cdot$ Lukasz Pakula $\cdot$ Izabela Zawlik
}

Received: 8 August 2013/Accepted: 11 January 2014/Published online: 29 January 2014

(C) The Author(s) 2014. This article is published with open access at Springerlink.com

\begin{abstract}
Triple negative breast cancer (TNBC) has caught the attention of oncologists worldwide because of poor prognosis and paucity of targeted therapies. Gene pathways have been widely studied, but less is known about epigenetic factors such as microRNAs (miRNAs) and their role in tailoring an individual systemic and surgical approach for breast cancer patients. The aim of the study was to examine selected miRNAs in TNBC core biopsies sampled before preoperative chemotherapy and the subsequent pathologic response in mastectomy or breast conservation specimens. Prior to treatment, core needle biopsies were collected from 11 female patients with inoperable locally advanced TNBC or large resectable tumors suitable for down-staging. In all 11 TNBC core
\end{abstract}

Agnieszka Kolacinska and Jan Morawiec contributed equally to this work.

A. Kolacinska $(\bowtie) \cdot$ J. Morawiec $\cdot$ Z. Morawiec

Department of Surgical Oncology, Copernicus Memorial

Hospital, Cancer Center, Paderewskiego 4, 93-509 Lodz, Poland

e-mail: info@drkolacinska.pl

J. Morawiec

Department of General and Colorectal Surgery, Medical

University of Lodz, Hallera Sq. 1, 90-647 Lodz, Poland

W. Fendler · B. Malachowska

Department of Pediatrics, Oncology, Hematology and

Diabetology, Medical University of Lodz, Sporna 36/50,

91-738 Lodz, Poland

J. Szemraj

Department of Medical Biochemistry, Medical University of Lodz, Mazowiecka 6/8, 92-215 Lodz, Poland

Z. Pawlowska

Central Laboratory Corelab, Medical University of Lodz,

Mazowiecka 6/8, 92-215 Lodz, Poland biopsies we analyzed 19 miRNAs per sample: 512, 190, 200, 346, 148, 449, 203, 577, 93, 126, 423, 129, 193, 182, 136, 135, 191, 122 and 222 (miRCURY LNA ${ }^{\mathrm{TM}}$ Universal RT microRNA polymerase chain reaction Custom Pick \& Mixpanels). The Wilcoxon signed-rank test was used to compare related samples. Ingenuity pathway analysis was used to evaluate potential functional significance of differentially expressed miRNAs. Statistical analysis showed that 3 of 19 miRNAs differed in relation to pathologic response i.e. good versus poor. These differences failed to reach statistical significance, although a trend was observed $(p=0.06)$. Among these miRNAs, we identified-miR200b-3p, miR-190a and miR-512-5p. In summary, our results indicate that higher miR-200b-3p, higher miR-190a and lower miR-512-5p expression levels in core biopsies sampled from TNBC patients may be associated with better pathologic response to chemotherapy and the increased

D. Chowdhury · Y. E. Choi

Department of Radiation Oncology, Dana-Farber Cancer

Institute, Harvard Medical School, 450 Brookline Av, Boston, MA 02215, USA

\section{R. Kubiak}

Department of Pathology, Medical University of Lodz,

Pabianicka 62, 93-513 Lodz, Poland

L. Pakula

Department of Anesthesiology, Copernicus Memorial Hospital, Pabianicka 62, 93-513 Lodz, Poland

\section{Zawlik}

Department of Medical Genetics, Institute of Nursing and Health Sciences, Medical Department, University of Rzeszow,

Rejtana 16c, 35-959 Rzeszow, Poland 
feasibility of breast conserving surgery in these patients. Although these results were from a small cohort, they provide an important basis for larger, prospective, multicenter studies to investigate the potential role of miRNAs in neoadjuvant setting.

Keywords Breast cancer - microRNA . Pathologic response

\section{Introduction}

Triple negative breast cancer (TNBC) has caught the attention of oncologists worldwide because of poor prognosis and paucity of targeted therapies [1]. The preoperative setting is an optimal model for in-depth research on TNBC $[2,3]$. Firstly there is the opportunity to fast-track testing of novel biomarkers and therapeutic agents, and secondly pathologic complete response (pCR) to neoadjuvant systemic therapy is a valid surrogate for better outcome in TNBC [4]. Multiple studies have demonstrated marked sensitivity of TNBC to chemotherapy compared with luminal A breast cancer [1-4]. Nevertheless 66-72 \% of TNBC patients fail to achieve pCR after treatment with preoperative anthracycline and taxane-based regimens, probably indicating adverse prognosis in terms of relapsefree and overall survival [4]. Oncologists are therefore pursuing more personalized therapies. Gene expression analysis has been widely incorporated in these studies, but less is known about epigenetic factors such as microRNAs (miRNAs) and their role in tailoring an individual systemic and surgical approach for breast cancer patients [5, 6]. MiRNAs are a regulatory class of small non-coding RNAs, approximately 20-23 nucleotides in length that have been described to post-transcriptionally modify gene expression. The deregulation of certain miRNAs has been associated with carcinogenesis in form of oncogenes, while others act as tumor suppressors [7].

\section{Aim}

The aim of the study was to examine selected miRNAs in TNBC core biopsies sampled before preoperative chemotherapy and the subsequent pathologic response in mastectomy or breast conservation specimens.

\section{Patients and methods}

The study was conducted under Institutional Review Board protocol \# RNN/226/11/KE/13/12/2011, Medical University of Lodz. All patients gave written informed consent.
Prior to treatment, ultrasound-guided 14-gauge core needle biopsies using an ultra automatic biopsy instrument (ProMag TM, Angiotech) were collected from 11 female patients with inoperable locally advanced breast cancer or large operable tumors suitable for down-staging, and from three healthy controls (breast reduction procedures) at the Cancer Center between December 2011 and April 2012. Four to five specimens were obtained from each lesion, half of which were frozen immediately at $-80{ }^{\circ} \mathrm{C}$, for subsequent miRNAs profiling. The other samples were paraffin embedded and reviewed by specialist breast pathologists in the Department of Pathology. Estrogen receptor (ER) and progesterone receptor (PR) status were determined by immunohistochemistry (IHC) using the Allred score.

Human epidermal growth factor receptor 2 (HER2) status was evaluated by IHC or by fluorescence in situ hybridization. Samples were considered ER/PR negative if less than $1 \%$ of the tumor cells were immunoreactive. Samples were considered HER2 negative with IHC $1+$ staining or with a score of $2+$ and no HER2 gene amplification when tested by FISH. TNBC were defined as ER, PR, HER2 negative. TNM clinical staging was assessed by mammography, ultrasound of the breast, axilla, and abdomen, bone scan and chest X-ray. In selected cases, MRI of the breast was performed. The following preoperative chemotherapy regimens were used: AT (doxorubicin $50 \mathrm{mg} / \mathrm{m}^{2}$, docetaxel $75 \mathrm{mg} / \mathrm{m}^{2}$ ) in five patients, AC (doxorubicin $60 \mathrm{mg} / \mathrm{m}^{2}$, cyclophosphamide $600 \mathrm{mg} / \mathrm{m}^{2}$ ) in four patients, EC (epirubicin $75 \mathrm{mg} / \mathrm{m}^{2}$, cyclophosphamide $600 \mathrm{mg} / \mathrm{m}^{2}$ )—in one patient and $\mathrm{AC}+\mathrm{T}$ (doxorubicin $60 \mathrm{mg} / \mathrm{m}^{2}$, cyclophosphamide $600 \mathrm{mg} / \mathrm{m}^{2}$, docetaxel $75 \mathrm{mg} / \mathrm{m}^{2}$ ) —in one patient. Upon completion of chemotherapy (six cycles every 21 days, except for $\mathrm{AC}+\mathrm{T}$ - eight cycles), specialist breast surgeons performed mastectomy or breast conservation, with axillary dissection or sentinel node biopsy. Pathologic response in the mastectomy or breast conservation specimens was assessed by specialist breast pathologists. pCR was defined as postoperative microscopic absence of invasive or in situ carcinoma in breast tissue, and axillary lymph nodes after preoperative chemotherapy. A near complete response with only minimal residual disease was described as small clusters of tumor cells in the primary tumor site or lymph node or minimal cellularity in the surgical specimen, with $90 \%$ loss of tumor cells. Pathologic non-response (pNR) was defined as no change or only minor change in individual malignant cells, but no reduction in overall cellularity. Partial pathologic response (pPR) was defined as reduction in overall cellularity, not exhibiting the changes listed for $\mathrm{pCR}$, near-pCR or pNR. pCR and near-pCR were key points in statistical analysis. 


\section{MiRNA profiling}

RNA was isolated using miReasy Mini Kit 50 (Qiagen). In all 11 TNBC core biopsies we analyzed 19 miRNAs per sample: hsa-miR-512-5p, hsa-miR-190a, hsa-miR-200b3p, hsa-miR-122-5p, hsa-miR-346, hsa-miR-148b-5p, hsamiR-449a, hsa-miR-191-5p, hsa-miR-203a, hsa-miR-577, hsa-miR-93-5p, hsa-miR-126-5p, hsa-miR-423-5p, hsamiR-129-5p, hsa-miR-193b-5p, hsa-miR-182-5p, hsa-miR136-5p, has-miR-222-5p and hsa-miR-135b-5p (Exiqon, Copenhagen, Denmark). We selected these miRNAs from the literature, miRNA database and breast cancer conferences. They represent various epigenetic pathways involved in migration, invasion, epithelial-mesenchymal transition, cancer dormancy, switch to the fast growing phenotype, drug resistance, etc. [8-18]. We also used unpublished information from miRNA in vitro profiling studies performed on breast cancer cell lines. For normalization of the data, we have applied the average of the hsamiR-103a-3p and hsa-miR-107 as this was found to be the most stable normalizer.

\section{MiRNA real-time qPCR}

10 ng RNA was reverse transcribed in $10 \mu \mathrm{l}$ reactions using the miRCURY LNA $^{\mathrm{TM}}$ Universal RT microRNA PCR, Polyadenylation and cDNA synthesis kit (Exiqon). Each RT was performed in duplicates. cDNA was diluted $100 \times$ and assayed in $10 \mu \mathrm{l}$ PCR reactions according to the protocol for miRCURY LNA ${ }^{\mathrm{TM}}$ Universal RT microRNA PCR; each microRNA was assayed once by qPCR on the microRNA Ready-to-Use PCR, Custom Pick-\&-Mix panel. Negative controls excluding template from the reverse transcription reaction were processes and profiled similarly. Amplification was performed in a LightCycler $^{\circledR} 480$ Real-Time PCR System (Roche, Basel, Switzerland) in 384-well plates. The amplification curves were analyzed using the Roche LC software, both for determination of $\mathrm{Cp}$ (by the second derivative method) and for melting curve analysis.

\section{Data analysis}

Amplification efficiency was calculated using algorithms similar to the LinReg software. All assays were inspected for distinct melting curves and the Tm was confirmed to be within known specifications for the assay. It was important for assays to be detected with $5 \mathrm{Cp}$ 's less than the negative control, and with $\mathrm{Cp}<37$ to be included in the data analysis. Data that failed to reach these criteria were excluded from further analysis. NormFinder was used to identify the optimum normalizer which was the average of assays detected in all samples (average - assay $\mathrm{Cp}$ ).

\section{Statistical analysis}

The Wilcoxon signed-rank test was used to compare related samples. As the study was considered preliminary and miRNAs tested were selected due to biologic function we did not adjust for multiple testing at this stage. Ingenuity pathway analysis (IPA) bioinformatic database was used to evaluate potential functional significance of differentially expressed miRNAs.

\section{Results}

Patients enrolled in the study were aged between 31- and 81-years-old, mean age was 54.1 years. Histopathological tumor types were invasive ductal breast cancer (11 patients). Tumor grades were G3 in all patients. Tumor stage was: IIB — in four patients, IIIA — in six patients, and IIIB - in one patient. Receptor status was triple negative in all patients. After preoperative chemotherapy, pCR was not achieved, but near-pCR was noted in $27 \%$ (3 out of 11) patients (Table 1). Statistical analysis showed that 3 of 19 miRNAs differed in relation to pathologic response. Among these miRNAs, we identified-miR-200b-3p ( $p=0.0662)$ up-regulated, miR-190a $(p=0.0662)$ upregulated and miR-512-5p $(p=0.0641)$ down-regulated.

$p$ values of analyzed miRNAs and type of pathologic response, e.g. near-pCR (good response) versus non-nearpCR (poor response) are shown in Table 2 and Fig. 1. The three miRNAs with $p$ values close to significance underwent pathway analysis using the IPA software. They were shown to be significantly linked to cellular assembly and organization/tissue development functional network with a $-\log 10(p)$ of nine representing a strong non-random association (Fig. 2).

\section{Discussion and conclusions}

In the current study, we hypothesized that altered levels of selected miRNAs correlated with sensitivity to preoperative chemotherapy in TNBC patients. Numerous studies have sought to identify miRNA markers in the serum of cancer patients, but little is known about how miRNAs levels found in breast cancer samples relate to drug resistance [8]. Tryndyak et al. [9] have shown convincingly that transfection of breast cancer cell line MDA-MB-231 with miRNA-200b-3p inhibits epithelial-to-mesenchymal transition (EMT), reversing this aggressive phenotype to a 
Table 1 Clinicopathological characteristics of breast cancer patients

\begin{tabular}{|c|c|c|c|c|c|c|c|c|c|}
\hline Patient & $\begin{array}{l}\text { Age } \\
\text { (years) }\end{array}$ & Race & $\begin{array}{l}\text { Histological } \\
\text { type }\end{array}$ & Grade & $\begin{array}{l}\text { Receptor } \\
\text { subtype }\end{array}$ & $\begin{array}{l}\text { Clinical staging before } \\
\text { preoperative } \\
\text { chemotherapy }\end{array}$ & $\begin{array}{l}\text { Preoperative } \\
\text { chemotherapy }\end{array}$ & $\begin{array}{l}\text { Pathological staging } \\
\text { in postoperative } \\
\text { specimen }\end{array}$ & $\begin{array}{l}\text { Type of } \\
\text { surgery }\end{array}$ \\
\hline Pt1 & 31 & White & IDC & G3 & $\mathrm{TN}$ & cT1N2 & AT & ypT1aN0 near-pCR & $\mathrm{BCS}$ \\
\hline Pt2 & 36 & White & IDC & G3 & $\mathrm{TN}$ & cT2N2 & $\mathrm{AC}+\mathrm{T}$ & $\begin{array}{l}\text { ypT0N2 pCR in } \\
\text { breast, no response } \\
\text { in axilla }\end{array}$ & Mastectomy \\
\hline Pt3 & 44 & White & IDC & G3 & $\mathrm{TN}$ & cT3N1 & AT & ypT1aN0 near-pCR & $\mathrm{BCS}$ \\
\hline Pt4 & 45 & White & IDC & G3 & $\mathrm{TN}$ & cT3N0 & $\mathrm{AC}$ & урT2N0 & Mastectomy \\
\hline Pt5 & 47 & White & IDC & G3 & $\mathrm{TN}$ & cT3N0 & $\mathrm{AC}$ & ypT2N0 & Mastectomy \\
\hline Pt6 & 55 & White & IDC & G3 & $\mathrm{TN}$ & cT3N1 & AT & ypT1aN0 near-pCR & BCS \\
\hline Pt7 & 55 & White & IDC & G3 & $\mathrm{TN}$ & cT2N1 & AT & ypT2N1 & Mastectomy \\
\hline Pt8 & 55 & White & IDC & G3 & $\mathrm{TN}$ & cT3N0 & $\mathrm{AC}$ & ypT2N0 & Mastectomy \\
\hline $\mathrm{Pt} 9$ & 66 & White & IDC & G3 & $\mathrm{TN}$ & cT4N1 & AT & урT3N3 & Mastectomy \\
\hline Pt10 & 80 & White & IDC & G3 & $\mathrm{TN}$ & cT2N2 & $\mathrm{EC}$ & ypT1cN1 & Mastectomy \\
\hline Pt11 & 81 & White & IDC & G3 & $\mathrm{TN}$ & cT3N1 & $\mathrm{AC}$ & ypT3N0 & Mastectomy \\
\hline
\end{tabular}

$I D C$ invasive ductal carcinoma, $T N$ triple negative

Preoperative chemotherapy regimens: $A$ doxorubicin, $E$ epirubicin, $C$ cyclophosphamide, $T$ docetaxel, $B C S$ breast conserving surgery

Table 2 Association of analyzed miRNAs and type of pathologic response (good vs. poor) in TNBC

\begin{tabular}{|c|c|c|c|c|c|}
\hline miRNA & Good response average $\mathrm{Cp}$ & SD poor response & Poor response average $\mathrm{Cp}$ & SD good response & $p$ value \\
\hline hsa-miR-512-5p & -11.27201 & 1.870254 & -8.702935 & 0.5255918 & 0.064078 \\
\hline hsa-miR-190a & -5.246738 & 0.9024946 & -6.213683 & 0.8006026 & 0.066193 \\
\hline hsa-miR-200b-3p & -1.997353 & 0.370737 & -2.960642 & 1.2972366 & 0.066193 \\
\hline hsa-miR-346 & -9.852577 & 1.1360037 & -9.914622 & 0.208277 & 0.182422 \\
\hline hsa-miR-148b-5p & -10.46267 & 0.5754232 & -10.71966 & 0.2461868 & 0.220671 \\
\hline hsa-miR-449a & -11.08681 & 0.6922827 & -11.00305 & 1.1720073 & 0.305059 \\
\hline hsa-miR-203a & -7.459372 & 1.0608366 & -6.144586 & 2.7639684 & 0.379775 \\
\hline hsa-miR-577 & -3.580436 & 0.3682042 & -3.571102 & 0.4376185 & 0.438578 \\
\hline hsa-miR-93-5p & -0.017407 & 0.9109349 & -0.546595 & 1.6692004 & 0.540291 \\
\hline hsa-miR-126-5p & -3.573845 & 1.4692401 & -3.6294 & 0.3382967 & 0.540291 \\
\hline hsa-miR-423-5p & -3.367891 & 0.6627491 & -3.099422 & 0.1176075 & 0.683091 \\
\hline hsa-miR-129-5p & -10.92072 & 0.9344205 & -10.62819 & 0.84145 & 0.698535 \\
\hline hsa-miR-193b-5p & -8.425986 & 0.6266225 & -7.939713 & 0.5464233 & 0.73244 \\
\hline hsa-miR-182-5p & -3.645599 & 1.0303241 & -3.417605 & 1.793051 & 0.838257 \\
\hline hsa-miR-136-5p & -4.099501 & 1.5175184 & -4.008906 & 0.1797492 & 0.838257 \\
\hline hsa-miR-135b-5p & -3.057166 & 1.638696 & -3.151112 & 1.4110264 & 0.838257 \\
\hline hsa-miR-191-5p & -2.948123 & 0.337603 & -3.021525 & 0.1843927 & 1 \\
\hline hsa-miR-122-5p & -12.42259 & 1.202344 & -12.26785 & 0.3695887 & 1 \\
\hline hsa-miR-222-5p & -11.04475 & 0.9186428 & -10.8786 & 1.0133855 & 1 \\
\hline
\end{tabular}

milder form and increasing sensitivity to doxorubicin. Similarly, we used preoperative anthracyclines in all patients with large tumors, and 3 out of 11 , with altered miRNA-200b-3p levels, responded well to treatment, with a tiny $2-\mathrm{mm}$ residual cluster of viable cancer cells in the primary tumor bed, permitting breast conservation. Perhaps in view of the small sample size, there was no statistically significant difference, although we observed a trend to significance $(p=0.06)$ for the hypothesized correlation. Further research is needed and the present study should be considered to be preliminary. Pogribny et al. [10] have pointed out that miR-200b-3p was among the most deregulated miRNAs in the MCF-7 cis-platin resistant human breast adenocarcinoma cell lines. In our study none 


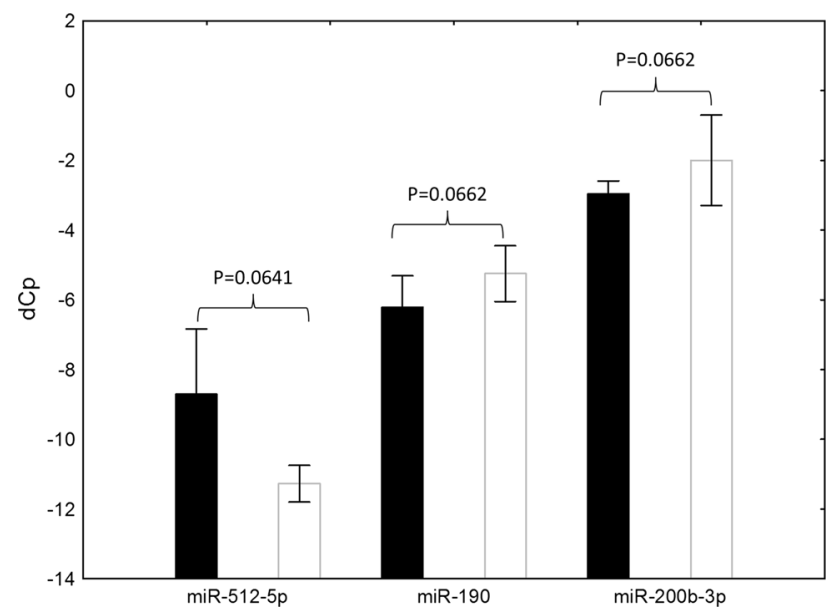

Fig. 1 Black bars represent patients with poor prognosis, while white bars represent miRNA expression levels of individuals with near-pCR (good prognosis) of the enrolled patients were treated with cis-platin, but based on the literature such regimens are efficacious in TNBC BRCA-mutation carrier breast cancer patients. However, in our study none of included patients were BRCA1/2 positive. Another miRNA whose expression differed between TNBC good and poor responders to anthracyclines and taxanes in our analysis was miR-190a ( $p=0.0662$, trend to significance).

Almog et al. have demonstrated in human breast carcinoma, glioblastoma, osteosarcoma and liposarcoma that over-expression of miRNA-190 governed the reverse switch of fast-growing angiogenic phenotype to the dormancy phase. These authors conducted their studies mainly in human cell lines, tissue cultures, mice and finally in 15 surgical specimens from patients with brain tumors [11, 12]. We have analyzed miR-190a expressions entirely in breast cancer patients, with reference to the
Fig. 2 Functional significance of differentially expressed miRNAs-miRNA-512-5p, 190a and 200b-3p (Ingenuity pathway analysis). Diamonds represent enzymes, triangles represent kinases, rectangles represent ion channels, trapezoid markers represent membrane transporters, inverted trapezoids represent miRNAs, circles represent other types of molecules. Arrows represent direction and type of interaction according to Ingenuity pathway analysis standard manual (http:// bioinfo.cnio.es/files/training/ Functional_Analysis_Course/ UBio_FuncAnalysis_Ingenuity. pdf)

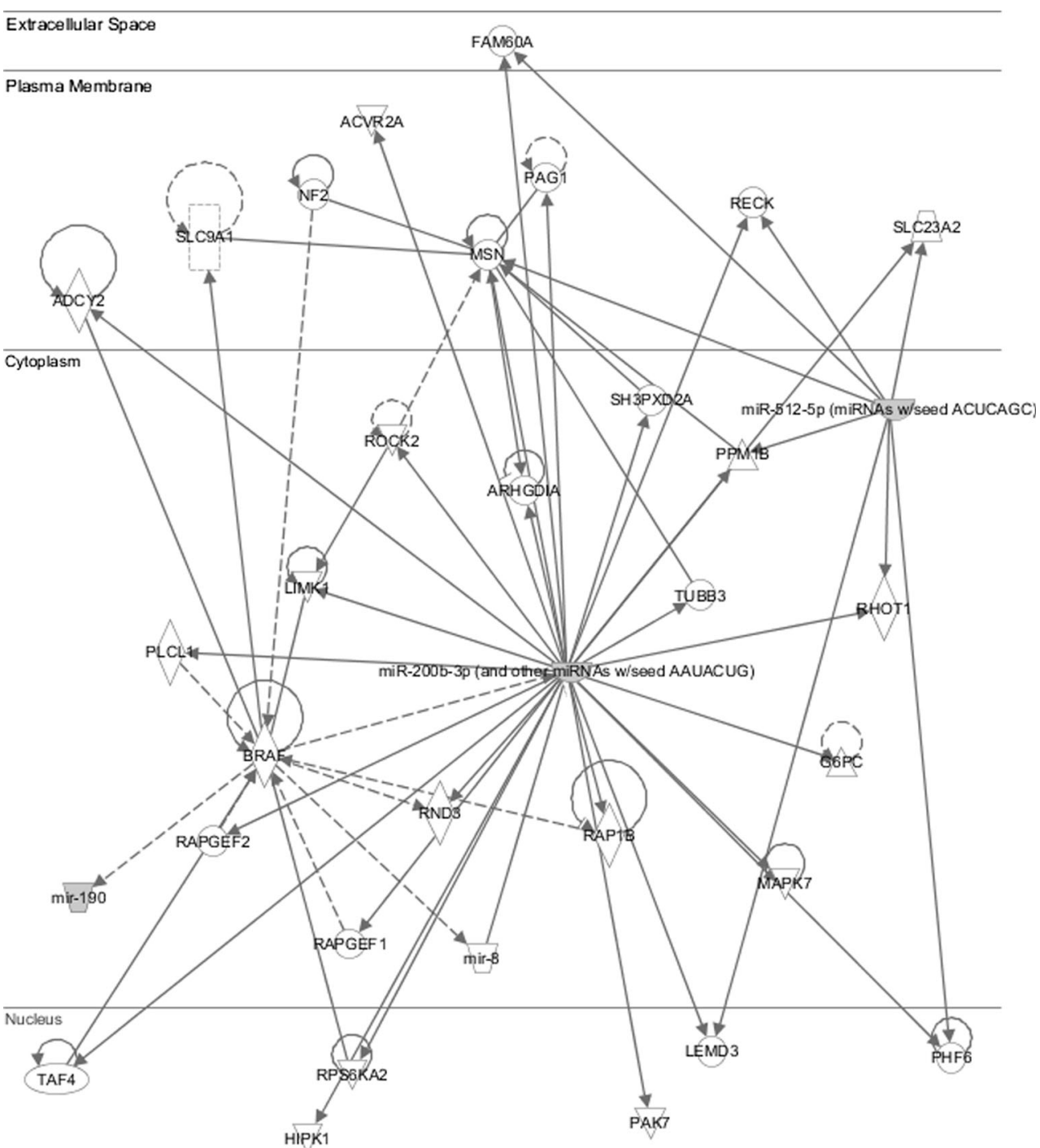


database of in vitro experimental models published by other researchers.

Port et al. [13], who compared miRNAs expression patterns (e.g., miR-512-5p) in chemoresistant cancer cell lines, have emphasized that it would be of interest to examine tumor samples of patients with both chemosensitive and chemoresistant tumors to analyze whether the fluctuations, e.g. up- and down-regulations of selected miRNAs, are also found in vivo.

Statistical analysis in the current in vivo study have shown differences in miR-512-5p expression in relation to pathologic response in TNBC patients that failed to reach statistical significance, although a trend was observed $(p=0.064)$. Further studies in larger cohorts are needed on account of the complexity of epigenetic modulations, their interactions with genetic pathways and the difficulty of direct translation from laboratory to clinic. We used two bioinformatic programs TargetScan and PicTar to identify target genes of miRNA 190a, 200b-3p and 512-5p; e.g. 104 target genes for miRNA 190a and 97 target genes for miRNA 200b-3p were found by PicTar, 94 target genes for miRNA 512-5p by TargetScan. Examples of target genes: BCL11A (B cell CLL/lymphoma 11A), CALCR (calcitonin receptor), FOXP2 (forkhead box P2), HOXC5 (homeobox C5) for miRNA 190a; PLCB1 (phospholipase C, beta 1), $M Y C N$ (v-myc myelocytomatosis viral related oncogene), CCND2 (cyclin D2), RERG (RAS-like, estrogen-related growth inhibitor) for miRNA 200b-3p; BCL2L2 (BCL2like 2), POLD3 polymerase (DNA-directed delta 3, accessory subunit), $c-M y c$ for miRNA 512-5p, etc. MiRNA and gene network is shown in Fig. 2 [12].

A key issue of neoadjuvant trials is pCR, as a surrogate end point, which strongly correlate with long-term survival in TNBC [4]. In our analysis none of 11 TNBC patients achieved pCR, but in three patients a good response to chemotherapy (near-pCR) was noted. Symmans et al. [14] have concluded in their study that minimal residual disease (RCB-I, residual cancer burden I according to M.D. Anderson criteria) in $17 \%$ of patients carried the same prognosis as pCR (RCB-0). Moreover, von Minckwitz et al. have demonstrated that Ki67 measured in residual hormone-receptor negative tumors can further subdivide this unfavorable group of patients without a pCR. High Ki67 levels in residual disease predict a considerable risk of relapse, but patients with low Ki67 levels showed a comparable outcome to patients with a pCR for diseasefree and overall survival [15]. In all three TNBC patients who achieved near-pCR in our study very low Ki67 levels (6\%) were measured.

In summary, our results indicate that higher miR-200b$3 p$, higher miR-190a and lower miR-512-5p expression levels in core biopsies sampled from TNBC patients may be associated with better pathologic response to chemotherapy and the increased feasibility of breast conserving surgery in these patients. Although these results were from a small cohort, they provide an important basis for larger, prospective, multicenter studies to investigate the potential role of miRNAs not only in breast cancer cells, but also in adjacent tissues and serum as predictive biomarkers. More precise identification, before initiation of treatment, of those patients who would benefit from specific chemotherapeutic regimens may improve response rates, avoid toxicity of ineffective therapy and guide the extent of necessary surgery i.e. breast conservation versus mastectomy [16-18].

Acknowledgments This study was supported by the grant of the Ministry of Science and Higher Education, Poland, 2011/01/B/NZ4/ 03345 .

\section{Conflict of interest None.}

Open Access This article is distributed under the terms of the Creative Commons Attribution License which permits any use, distribution, and reproduction in any medium, provided the original author(s) and the source are credited.

\section{References}

1. Piccart MJ (2012) Triple-negative breast cancer in focus: from biology to novel therapeutics. Editorial. Ann Oncol 23(Suppl 6):vi5-6

2. Von Minckwitz G, Martin M (2012) Neoadjuvant treatments for triple negative breast cancer. Ann Oncol 23(Suppl 6):vi5-6

3. Von Minckwitz G, Untch M, Loibl S (2013) Update on neoadjuvant/preoperative therapy of breast cancer: experiences from the German Breast Group. Curr Opin Obstet Gynecol 25:66-73

4. Von Minckwitz G, Untch M, Biohmer JU, Costa SD, Eidtmann H et al (2012) Definition and impact of pathologic complete response on prognosis after neoadjuvant chemotherapy in various intrinsic breast cancer subtypes. J Clin Oncol 30:1796-1804

5. Ellis MJ, Perou CM (2013) The genomic landscape of breast cancer as a therapeutic roadmap. Cancer Discov 3:27-34

6. Balic M, Schwarzenbacher D, Stanzer S, Heitzer E, Auer M et al (2013) Genetic and epigenetic analysis of putative breast cancer stem cell models. BMC Cancer 13:358. doi:10.1186/1471-240713-358

7. Dvinge H, Git A, Graf S, Salmon-Divon M, Curtis C et al (2013) The shaping and functional consequences of the microRNA landscapes in breast cancer. Nature 497:378-382

8. Jung EJ, Santarpia L, Kim J, Esteva FJ, Moretti E et al (2012) Plasma microRNA 210 levels correlate with sensitivity to trastuzumab and tumor presence in breast cancer patients. Cancer 118:2603-2614

9. Tryndyak VP, Brland FA, Pogribny IP (2010) E-cadherin transcriptional down-regulation by epigenetic and microRNA-200 family alterations is related to mesenchymal and drug-resistant phenotype in human breast cancer cells. Int J Cancer 126:2575-2583

10. Pogribny IP, Filkowski JN, Tryndyak VP, Golubov A, Shpyleva SI, Kovalchuk O (2010) Alterations of microRNAs and their targets are associated with acquired resistance of MCF-7 breast cancer cells to cisplatin. Int J Cancer 127:1785-1794

11. Almog N, Ma L, Schwager C, Brinkmann BG, Beheshti A, Vajkoczy P (2012) Consensus microRNAs governing the switch of 
dormant tumors to the fast-growing angiogenic phenotype. PLoS ONE 7:e44001. doi:10.1371/journal.pone.0044001

12. Almog N, Briggs C, Beheshti A, Ma L, Wilkie KP et al (2013) Transcriptional changes induced by the tumor dormancy-associated microRNA 190. Transcription 4 [Epub ahead of print]

13. Port M, Glaesener S, Ruf C, Riecke A, Bokemeyer C et al (2011) Micro-RNA expression in cisplatin resistant germ cell tumor cell lines. Mol Cancer 10:52. doi:10.1186/1476-4598-10-52

14. Symmans WF, Peintinger F, Hatzis C, Rajan R, Kuerer H et al (2007) Measurement of residual breast cancer burden to predict survival after neoadjuvant chemotherapy. J Clin Oncol 25:4414-4422

15. Von Minckwitz G, Schmitt W, Loibl S, Muller BM, Blohmer JU et al (2013) Ki67 measured after neoadjuvant chemotherapy for primary breast cancer. Clin Cancer Res. doi:10.1158/1078-0432. CCR-12-3628

16. Sun L, Yao Y, Lin B, Lin Z, Lin L et al (2012) MiR-200b and miR-15b regulate chemotherapy-induced epithelial-mesenchymal transition in human tongue cancer cells by targeting BMI1. Oncogene 31:432-445

17. Rutnam ZJ, Yang BB (2012) The non-coding $3^{\prime}$ UTR of CD44 induces metastasis by regulating extracellular matrix functions. J Cell Sci 125:2075-2085

18. Chan M, Liaw CS, Ji SM, Tan HH, Wong CY et al (2013) Identification of circulating microRNA signatures for breast cancer detection. Clin Cancer Res. doi:10.1158/1078-0432.CCR-12-3401 